\title{
Active Integrated Tracking Detectors for MRI-Guided Interventions
}

J. Anders, University of Ulm, Department of Engineering, Ulm, Germany, jens.anders@uni-ulm.de M. Ortmanns, University of Ulm, Department of Engineering, Ulm, Germany, maurits.ortmanns@uni-ulm.de K. Scheffler, Max-Planck-Institute for Biological Cybernetics, Germany, Tübingen, klaus.scheffler@tuebingen.mpg.de G. Boero, Ecole Polytechnique Fédérale de Lausanne, Department of Engineering, Lausanne, Switzerland, giovanni.boero@epfl.ch

\section{Introduction}

Thanks to its three-dimensional imaging capabilities with excellent soft-tissue contrast, magnetic resonance imaging is gaining interest as a tool for guided interventions. All active tracking detectors currently used for the positioning of interventional devices during MRI-guided procedures use hand-wound detection coils with external electronics.

\section{Methods}

We use standard CMOS technology to manufacture fully-integrated NMR magnetometer chips which can be attached to interventional devices in order to determine their position during MRI-guided interventions. The chips co-integrate an NMR detection coil and a complete downconversion receiver on the same die. The detectors can work with dedicated, permanent samples or can utilize the chip environment as sample. Then, knowing the applied gradient strength and measuring the local Larmor frequency it is possible to determine the chip position and thereby also the position of the interventional device it is attached to.

\section{Results}

We have characterized a fully-integrated CMOS tracking detector with a size of $1 \times 1.9 \times 0.8 \mathrm{~mm}^{3}$, which can be used with medium-size catheters. The design has been successfully interfaced with a standard clinical scanner and achieves an isotropic spatial resolution of $0.15 \mathrm{~mm}$ in a measuring time of $100 \mathrm{~ms}$.

\section{Conclusion and outlook}

We have presented and previously published [1] a fully-integrated tracking detector with a state-of-the-art performance in terms of spatial resolution. Currently, we are designing an updated version of the previous chip with a reduced size to make it suitable with the use in smaller size catheters. Apart from their high-reproducibility, and low manufacturing costs for large-volume fabrication one of the biggest advantages of the fully-integrated tracking devices is their on-chip LO generation and frequency downconversion which removes the need for high-frequency connections through the human body and thereby allows for an effective filtering of signals induced in cables during excitation.

1. Anders, J., et al., Integrated active tracking detector for MRI-guided interventions. Magnetic Resonance in Medicine, 2012. 67(1): p. 290-296. 